\title{
ESTIMACIÓN INDIRECTA DEL ÁREA FOLIAR EN Fragaria vesca L., Physalis peruviana L., Acca sellowiana (Berg.) Burret, Rubus glaucus L., Passiflora mollissima (Kunth) L. H. Bailey Y Ficus carica L
}

\author{
INDIRECT ESTIMATION OF LEAF AREA IN Fragaria \\ vesca L., Physalis peruviana L, Acca sellowiana \\ (Berg.) Burret, Rubus glaucus L., Passiflora \\ mollissima (Kunth) L. H. Bailey AND Ficus carica L.
}

Fánor Casierra-Posada ${ }^{1}$ Germán Ricardo Peña Z. ${ }^{2}$ Jaime E. Peña-Olmos ${ }^{3}$

\section{RESUMEN}

La estimación rápida, objetiva y confiable del área foliar es esencial en numerosos estudios de fisiología vegetal; sin embargo, Ésta se realiza usualmente mediante métodos destructivos o depende de la disponibilidad de medidores electrónicos integrados, costosos y sofisticados. En esta investigación, se calculó la ecuación más confiable para predecir el área foliar, en seis especies frutícolas cultivadas en Boyacá, Colombia, a través de mediciones lineales de la geometría de la hoja. Las especies evaluadas fueron fresa, uchuva, feijoa, mora, curuba y brevo. Las ecuaciones, se calcularon con mediciones de la longitud y ancho de la hoja y del área foliar de 500 hojas, de cada especie, a través de un análisis de regresión. Posteriormente, las ecuaciones halladas, se comprobaron aplicándolas a

${ }^{1}$ Ingeniero Agrónomo PhD., profesor asociado en la Facultad de Ciencias Agropecuarias de la Universidad Pedagógica y Tecnológica de Colombia. Apartado aéreo 661, Tunja - Boyacá, Colombia. Grupo de Investigación Ecofisiología Vegetal. E-mail: fanor.casierra@uptc.edu. co [Autor para correspondencia]

2 Ingeniero Agrónomo, Universidad Pedagógica y Tecnológica de Colombia, Tunja - Boyacá. E-mail: geripeza1978@yahoo.es

3 Ingeniero Agrónomo, Universidad Pedagógica y Tecnológica de Colombia, Tunja - Boyacá. E-mail: jepo8503@yahoo.es
100 hojas, en las cuales se había medido el área foliar. Con la información observada y estimada, se realizó una prueba de correlación de Pearson. Las ecuaciones utilizaban, como variable, el producto de la longitud de la hoja por el ancho máximo de la misma y presentaron alta correlación con el área foliar observada, manifestada en coeficientes de determinación altos $\left(R^{2}=0,99\right)$, en las ecuaciones y, error estándar bajo, en los datos estimados. Los coeficientes de correlación de Pearson entre el área foliar observada y estimada estuvo en el rango de 0,950,99, indicando significancia al nivel de 0,01 para todas las especies. Por tanto, el uso del producto de la longitud por el ancho de la hoja, como variable, fue muy acorde para predecir el área foliar en las especies evaluadas.

Palabras clave: Fresa, uchuva, feijoa, mora, curuba, brevo.

\section{SUMMARY}

Rapid, reliable and objective estimation of leaf area is essential for numerous studies in plant physiology, however, it is done usually by means of destructive methods or it depends on the availability of sophisticated and expensive electronic integrate area meters. In this research, the most reliable equation that predicts the leaf area of six fruit species growing in Boyacá, Colombia, by non-destructively linear measurements of leaf geometry was calculated. Evaluated species were strawberry, cape 
gooseberry, pineapple guava, blackberry, banana passion fruit and common fig. The equations were developed by measuring lamina width, length and leaf area of 500 leaves of each species, by means of a regression analysis. Then, the calculated equations were confirmed, by their application to 100 leaves, of which the leaf area had been measured. With the observed and estimated information a Pearson correlation analysis was realized. Equations which used as variable the product of the leaf length and its maximum width presented strong relationships with the observed leaf area, manifested in high determination coefficients $\left(R^{2}=0,99\right)$ of the equations and low standard error of the estimated data. The Pearson correlation coefficients were between 0.95-0.99 for all species, indicating significance at 0.01. Therefore, the use of the product of leaf length by width as the variable was found to be accurate to predict the leaf area for all evaluated fruit species.

Key words: Strawberry, cape gooseberry, pineapple guava, blackberry, banana passion fruit, common fig.

\section{INTRODUCCIÓN}

La fruticultura colombiana es rica en especies y materiales genéticos que permiten a los cultivadores incursionar en los mercados con productos frescos, por la gran variedad de climas que se presentan en el territorio nacional y la particular topografía del país. La curuba, la mora, la feijoa y la uchuva, se han identificado como especies promisorias (Ministerio de Agricultura y Desarrollo Rural, 2004). Adicionalmente, el brevo y la fresa tienen una importancia local en los departamentos de Cundinamarca, Cauca, Norte de Santander y Boyacá, dado que las condiciones agroclimáticas hacen posible la obtención de volúmenes de fruta, económicamente rentables. Por estas razones, se hace indispensable realizar investigaciones con estas especies vegetales promisorias, orientadas a la determinación de las variables, involucradas en el rendimiento, entre las cuales, el área foliar juega un papel de gran relevancia.

Las hojas constituyen el órgano aéreo más importante en la planta, el cual tiene un papel determinante en las actividades metabólicas, en las que participan las clorofilas, que se encuentran en abundancia en ellas. Estos pigmentos son básicos en el proceso fotosintético que, a su vez, es uno de los mayores procesos de síntesis en los vegetales. De ahí, que el área foliar total que, en la mayoría de los casos tiene una relación directa con la cantidad de clorofila, es un parámetro importante para determinar la posibilidad de la planta para sintetizar su materia seca (Prasada Rao et al. 1978). Por estas razones, se ha propuesto que las mediciones en el incremento y la duración del área foliar ofrecen un método alternativo de interés en la determinación del potencial fotosintético de la corona de las plantas (Landsberg, 1986; Landsberg $\mathcal{E}$ Hingston, 1996).

Estudios recientes relacionados con la obtención de modelos de interacción entre la dinámica de los regímenes de radiación y el desarrollo del dosel de las plantas, se han orientado a la intercepción de la energía incidente, como un parámetro para la predicción de la producción de materia seca (Kozlowski et al. 1991). En algunas localidades donde las condiciones ambientales son favorables para la fotosíntesis, la cantidad de superficie fotosintética producida y su eficiencia en la captura de luz son los responsables de las variaciones en el rendimiento agronómico (Cannell et al. 1997; Linder et al. 1985).

El área foliar es una variable importante en la mayoría de los estudios agrícolas y fisiológicos involucrados en el crecimiento vegetal, captación de luz, eficiencia fotosintética, respiración, transpiración y respuesta al riego y a la fertilización. La determinación del área foliar, se realiza generalmente mediante métodos directos, en los cuales las hojas tomadas de las plantas en los experimentos, se analizan con la ayuda de un medidor de área electrónico integrado. Éste, además de ser un método destructivo, se restringe a la disponibilidad del equipo. Los métodos indirectos son de gran utilidad cuando no se dispone de equipos especializados, cuando se necesita realizar mediciones no destructivas o para llevar a cabo estudios con especies vegetales nativas, que crecen lejos de centros de investigación o universidades que poseen los equipos necesarios (Blanco E Folegatti, 2005).

Adicionalmente, se ha utilizado la lectura de las hojas mediante un escáner convencional, con el propósito de determinar tanto el área foliar como los niveles de defoliación, lo cual resulta en un método económico y preciso (O’Neal et al. 2002).

En el desarrollo de estrategias de protección de cultivos contra plagas insectiles, se han establecido modelos geométricos, en los cuales la estimación del área foliar destruido por insectos comedores del follaje es de suma 
importancia para definir niveles de daño, que requieren un control para evitar que causen pérdidas económicas (Ebert \& Derksen, 2004).

Uno de los métodos no destructivos más utilizados es la estimación del área foliar a partir de ecuaciones matemáticas, que involucran mediciones de largo y ancho de las hojas. Esta herramienta ha mostrado gran exactitud en plantas de lechuga, Lactuca sativa (Guo \& Sun, 2001) y tomate, Lycopersicon esculentum (Astegiano et al. 2001).

El área foliar, se puede medir mediante el uso de instrumentos o con la implementación de modelos de predicción. Sin embargo, la determinación del área foliar mediante instrumentos puede ser dispendiosa en los casos de hojas con formas complejas, relacionadas con el número y tamaño de las nervaduras y con la geometría de los bordes (Campostrini E Yamanishi, 2001). Recientemente, se han desarrollado nuevas herramientas y equipos, tales como escáneres manuales y equipos ópticos de lectura por láser, que tienen la posibilidad de realizar mediciones muy precisas en hojas y frutos. Como alternativa, la estimación del área foliar, se puede realizar utilizando mediciones geométricas en las hojas, como variables, en ecuaciones (Robbins \& Pharr, 1987).

En la actualidad, la explotación mejorada de las especies vegetales maderables y el creciente interés por la estructura y la función de los ecosistemas forestales ha estimulado la necesidad de estimaciones precisas de la producción de biomasa y del área foliar de las especies arbóreas. Se usan, comúnmente, ecuaciones de regresión para predecir la producción de biomasa, a partir de parámetros de fácil medición (Halpern et al. 1996; Ola-Adams, 1997). Se han utilizado ecuaciones alométricas para estimar la biomasa y el área foliar en varios tipos de plantas (Burton et al. 1991; Lövenstein E Berliner, 1993; Lavigne et al. 1996). La forma de las ecuaciones alométricas varia ampliamente, pero la más utilizada es la que está en función del logaritmo natural, $[y=a+b \ln (d b h)]$, en donde [y] es la biomasa ó el área foliar y $[d b h]$ corresponde al diámetro del árbol a la altura del pecho (Lin et al. 2001).

El objetivo del estudio fue la determinación de las ecuaciones de regresión más acertadas para la estimación no destructiva del área foliar en plantas de fresa, uchuva, feijoa, mora, curuba y brevo, con miras a encontrar y divulgar una herramienta útil en los estudios de fisiología vegetal, entomología y de rendimiento agronómico en estos cultivos.

\section{MATERIALES Y MÉTODOS}

El estudio, se realizó en el laboratorio de fisiología vegetal de la Facultad de Ciencias Agropecuarias de la Universidad Pedagógica y Tecnológica de Colombia, en Tunja. Para el desarrollo del trabajo, se tomaron 500 hojas adultas y sanas, de cada especie, las cuales estaban completamente expandidas. Los frutales, motivo de estudio, fueron fresa, uchuva, feijoa, mora, curuba y brevo, que se encontraban en la etapa productiva, en los cuales se midió el área foliar, con un medidor integrado Li-cor 3000a (USA), así como el largo de la lámina foliar y el ancho máximo de la misma, con la ayuda de un nonio. Con la información colectada, se realizó un análisis de regresión y se calcularon las ecuaciones, de las cuales, se seleccionó el modelo de ecuación, que presentara el coeficiente de determinación más alto. Como variable independiente en la ecuación, se tomó el producto del largo por el ancho de la lámina foliar, en centímetros; sin embargo, en el caso de las hojas de fresa, se tomó como variable para la ecuación la sumatoria del largo (cm) de cada uno de los foliolos de la hoja compuesta por la sumatoria de los anchos, dado que los foliolos presentan formas relativamente irregulares. En el caso de mora, a pesar de que la hoja es compuesta y presenta tres foliolos, cada uno se tomó como una hoja independiente, de manera que la variable para la ecuación fue el producto, en centímetros, del ancho por el largo de un foliolo, dado que los mismos presentan forma bastante regular, como lo muestra la figura 1 .

Posteriormente, se tomaron del campo 100 hojas más de cada especie frutícola, a la cuales se midió el área foliar, el largo y el ancho de la hoja y se validaron las ecuaciones calculadas con anterioridad. Se realizó una prueba de correlación de Pearson entre el área foliar observada con el medidor integrado y la calculada con la ecuación. Tanto los cálculos de regresión, como de correlación, se realizaron con la aplicación SPSS (Statistical Product and Service Solutions) versión 11.5.1 (nov. 2002).

\section{RESULTADOS Y DISCUSIÓN}

En todas las especies evaluadas, se encontró que el modelo cúbico de la ecuación de regresión fue el más ajustado para la estimación del área foliar, lo que fue confirmado por el alto coeficiente de determinación 
encontrado $\left(R^{2}=0,99\right)$. Las ecuaciones calculadas para cada caso, se encuentran listadas en la tabla 1. Además, en todos los casos, el modelo presentó alta significancia al nivel de 0,01.
Se observó gran concordancia entre la información obtenida para el área foliar estimada y observada, como se muestra en la figura 2. En el caso de las hojas de brevo y de curuba, los datos estuvieron más dispersos a

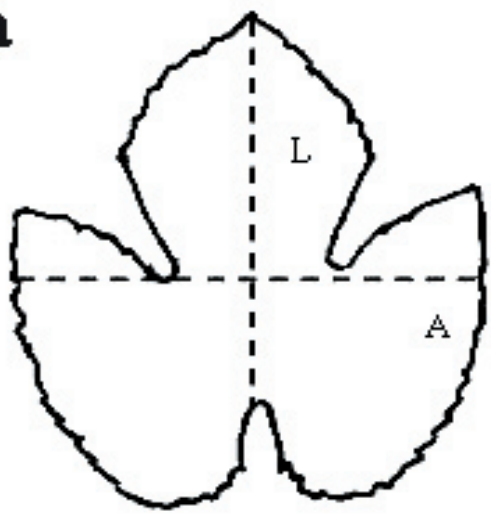

c
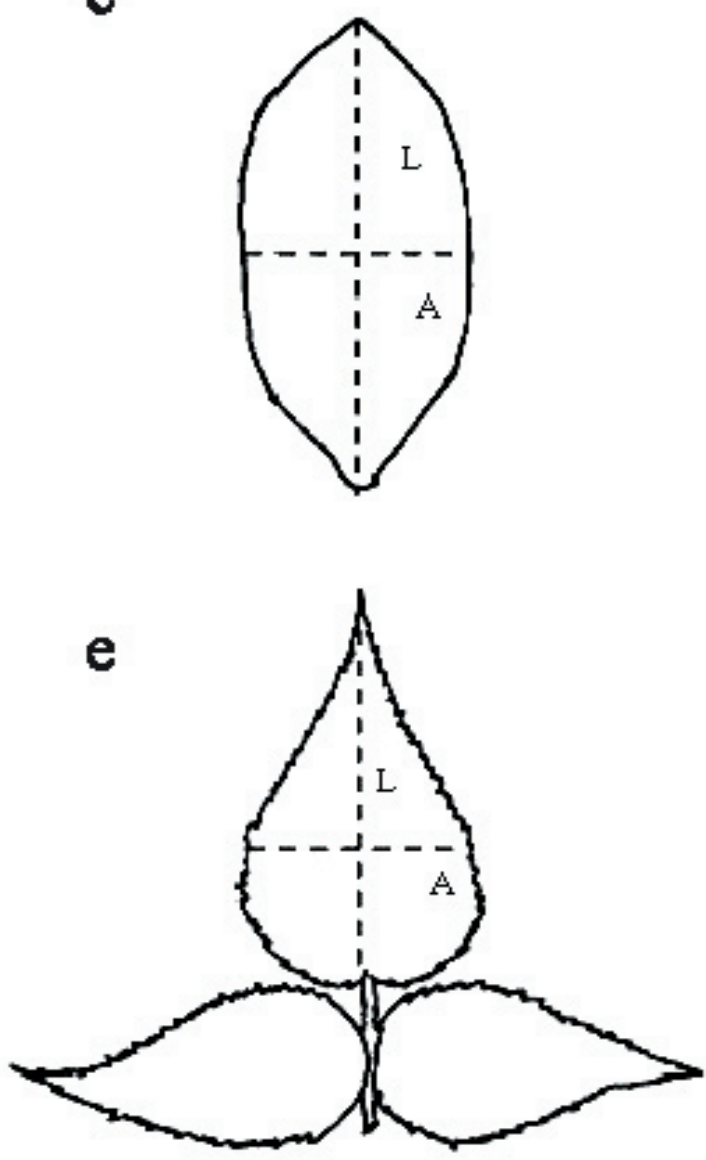

b

b

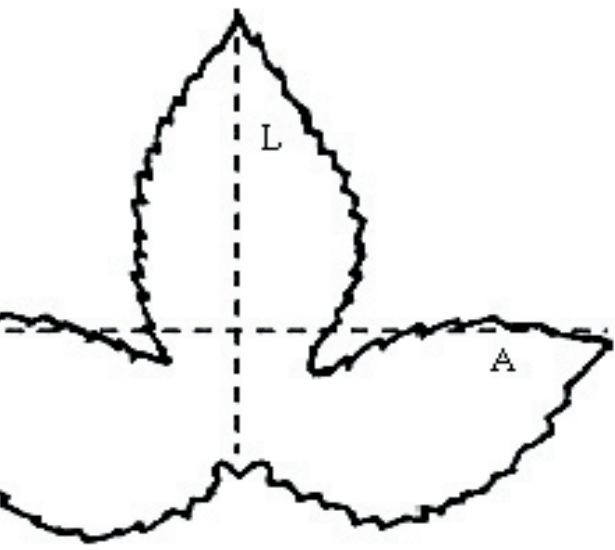

d

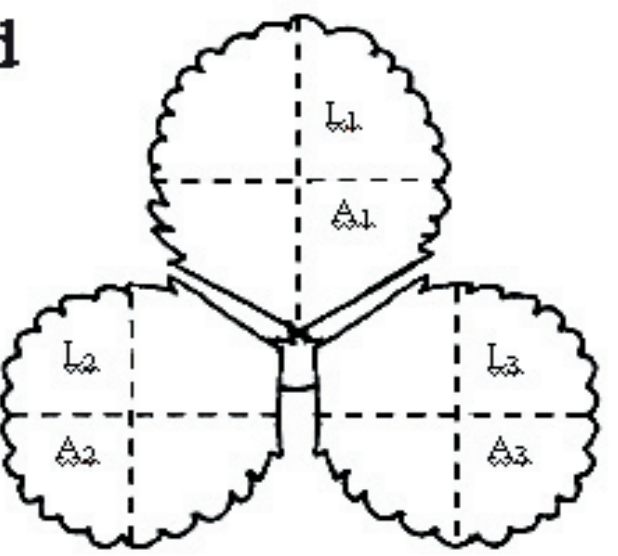

f

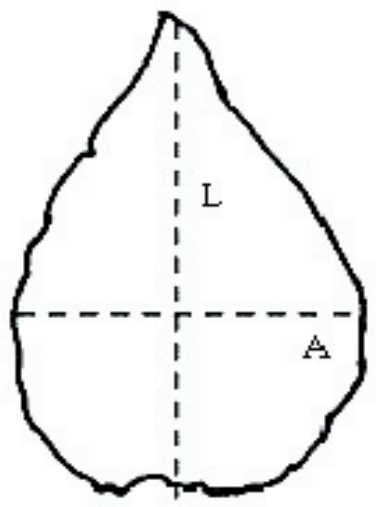

Figura 1. Diagrama de la toma de las variables largo (L) y ancho (A) en hojas de: a) brevo; b) curuba; c) feijoa; d) fresa; e) mora y f) uchuva. 
Tabla 1. Ecuaciones de regresión para estimar el área foliar en seis especies frutícolas, con base en el largo y el ancho de las hojas.

\begin{tabular}{|c|l|l|}
\hline Especie & \multicolumn{1}{|c|}{ Ecuación de regresión } & $\mathbf{R}^{2}$ \\
\hline Brevo & $y=0,96354 \mathrm{x}-0,000739 \mathrm{x}^{2}+8,58601011 \cdot 10^{-7} \mathrm{x}^{3}$ & $0,99^{* *}$ \\
\hline Curuba & $\mathrm{y}=0,596944 \mathrm{x}-0,000821 \mathrm{x}^{2}+1,49412692 \cdot 10^{-6} \mathrm{x}^{3}$ & $0,99^{* *}$ \\
\hline Feijoa & $\mathrm{y}=0,868539 \mathrm{x}-0,007734 \mathrm{x}^{2}+0,000111 \mathrm{x}^{3}$ & $0,99^{* *}$ \\
\hline Fresa & $\mathrm{y}=0,286141 \mathrm{x}-8,5624061 \cdot 10^{-5} \mathrm{x}^{2}-1,06547953 \cdot 10^{-8} \mathrm{x}^{3}$ & $0,99^{* *}$ \\
\hline Mora & $\mathrm{y}=0,637859 \mathrm{x}+0,000146 \mathrm{x}^{2}-1,875055 \cdot 10^{-6} \mathrm{x}^{3}$ & $0,99^{* *}$ \\
\hline Uchuva & $\mathrm{y}=0,728545 \mathrm{x}-0,001184 \mathrm{x}^{2}+8,60036002 \cdot 10^{-6} \mathrm{x}^{3}$ & $0,99^{* *}$ \\
\hline
\end{tabular}

** Significativo al nivel de 0,01

$\mathrm{y}=$ área foliar estimada $\left(\mathrm{cm}^{2}\right)$

$\mathrm{x}=$ producto del largo por el ancho máximo de la hoja $(\mathrm{cm})$

estadísticamente que para las demás especies, lo cual se debe a que las hojas de estas plantas presentan bordes accidentados, lo que dificulta su medición, como lo describen Campostrini E Yamanishi (2001). Los datos observados y calculados para estas dos especies presentaron la mayor dispersión, entre las especies evaluadas, pues las hojas muestran limbo en forma lobulada, lo que puede inducir a errores en la determinación del ancho de la lámina foliar. La información calculada para las hojas de uchuva presentó el mayor índice de ajuste con los datos observados, posiblemente, debido a que las hojas de esta especie tienen forma de la lámina foliar más uniforme que la exhibida por las demás especies, lo que se evidenció en una menor dispersión de los datos.

Otros autores reportan también alta concordancia entre el área foliar y la geometría de la hoja expresada en términos de largo y de ancho, en varias plantas cultivadas, como Fragaria sp. (Strik \& Proctor, 1985), Carthamus tinctorius (Sepaskhah, 1977), Lactuca sativa (Guo \& Sun, 2001) y Colocasia esculenta (Lu et al. 2004), Phaseolus vulgaris (Warnock et al. 2006). A pesar de que las mediciones requieren más tiempo para la determinación del área foliar por la toma de datos del largo y del ancho, éstos son los parámetros comúnmente utilizados, debido a la alta precisión en los resultados y el bajo error en las predicciones (Blanco \& Folegatti, 2005).

Respecto a la validación de las ecuaciones calculadas, se observó, mediante los resultados de la prueba de correlación de Pearson, que había gran concordancia entre los datos observados y los calculados con las ecuaciones respectivas para cada especie (Tabla 2). En todos los casos, se presentó significancía al nivel de 0,01.

Tabla 2. Coeficiente de correlación de Pearson entre área foliar observada y estimada en seis especies frutícolas.

\begin{tabular}{|c|c|}
\hline Especie evaluada & Coeficiente de correlación de Pearson \\
\hline Brevo & $0,952^{* *}$ \\
\hline Curuba & $0,953^{* *}$ \\
\hline Feijoa & $0,963^{* *}$ \\
\hline Fresa & $0,959^{* *}$ \\
\hline Mora & $0,973^{* *}$ \\
\hline Uchuva & $0,994^{* *}$ \\
\hline
\end{tabular}

** La correlación es significativa al nivel 0,01 

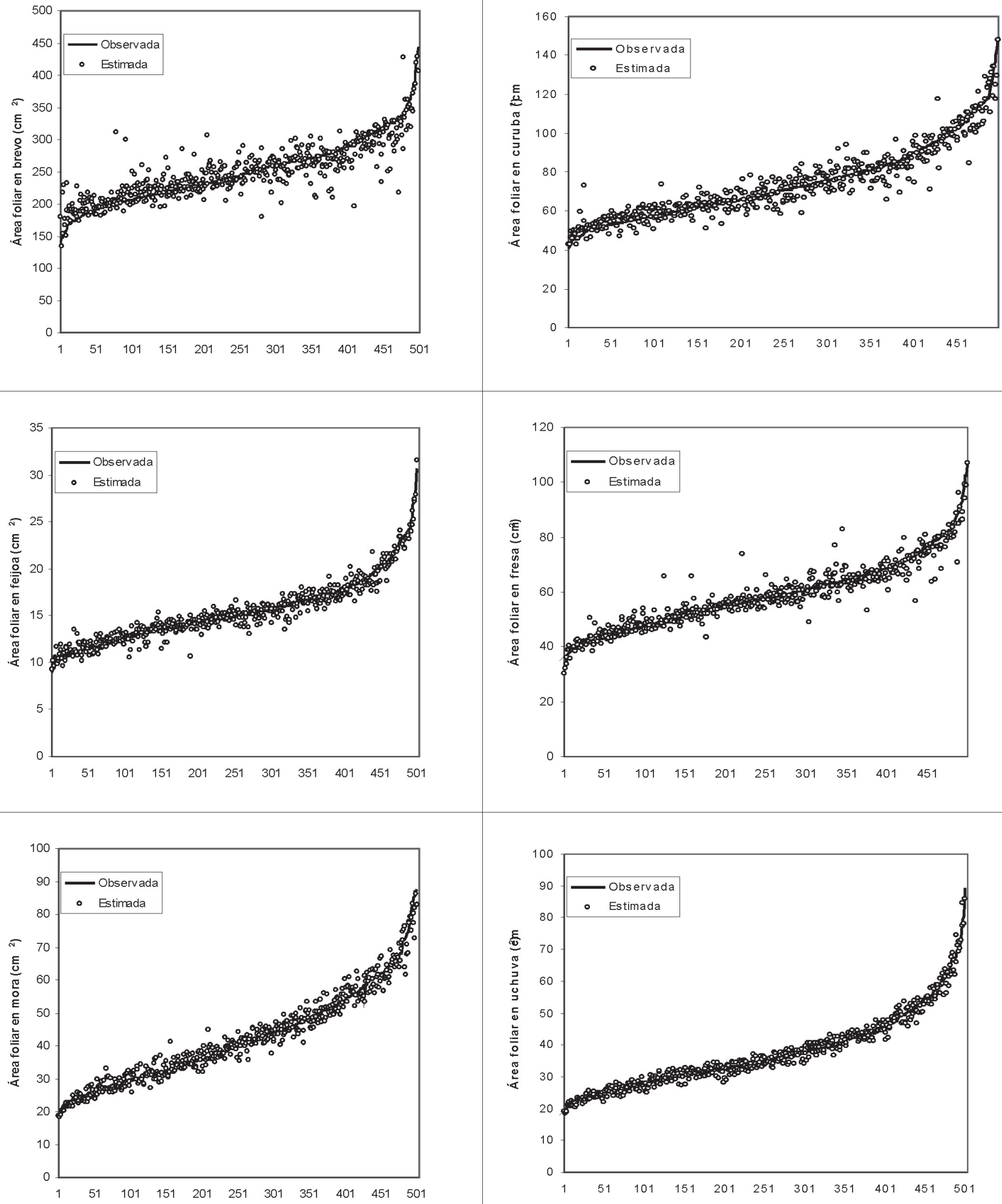

\section{Número de observaciones}

Figura 2. Área foliar observada y calculada en seis especies frutícolas. 
La validación de los modelos obtenidos para la predicción del área foliar es un paso importante para evaluar el grado de precisión de los modelos obtenidos. La confiabilidad en la precisión de los modelos proporciona a los investigadores un método relativamente rápido y económico que se puede utilizar como complemento en estudios de fisiología vegetal. Los coeficientes de correlación más bajos, se presentaron para brevo, curuba y fresa, por las razones antes expuestas, relacionadas con la forma de las hojas, a pesar de lo cual, se consideran coeficientes que ofrecen alto grado de confiabilidad, por estar por encima de 0,90. Debido a la uniformidad en la forma de las hojas de uchuva es de nuevo esta planta la que mostró el mayor coeficiente de correlación entre los datos obtenidos con el medidor integrado y los calculados con la ecuación encontrada.

El parámetro comúnmente utilizado en la validación de las ecuaciones encontradas es el coeficiente de determinación $\left(R^{2}\right)$. Al respecto, Çelik \& Uzun (2002) encontraron valores de $\mathrm{R}^{2}$ del orden de 0,988 para mediciones directas y estimadas de área foliar en Piper nigrum y, por su parte, Demrsoy et al. (2005) realizaron la validación de la información directa e indirecta de área foliar en Fragaria sp. por un valor de $\mathrm{R}^{2}$, el cual estuvo alrededor de 0,99, con lo cual demostraron la utilidad y la confiabilidad de los modelos calculados en varios cultivares de fresa, mantenidos bajo diferentes ambientes.

En el presente estudio, a pesar de que se calculó el coeficiente de determinación $\left(R^{2}\right)$ y se encontró que en todos las especies evaluadas su valor fue de 0,99 , se consideró necesaria la validación de la aplicación de los modelos comparándolos con los datos de mediciones directas en las mismas hojas y, según los coeficientes de correlación, se comprobó el grado de acierto de los modelos; por tanto, las ecuaciones reportadas para cada una de las especies evaluadas presentan alto grado de confiabilidad en la determinación del área foliar, mediante estimaciones no destructivas, tomando como variables de entrada el largo y el ancho de las hojas.

\section{CONCLUSIONES}

Las ecuaciones de regresión encontradas se ajustan en gran medida para la determinación indirecta del área foliar, tomando como parámetros de entrada la longitud y el ancho de las hojas, en las seis especies evaluadas. El largo y el ancho de las hojas son valores confiables que, aplicados a las ecuaciones de regresión encontradas, expresan con una exactitud entre 95 y 99\% el área foliar en las especies sometidas a evaluación.

\section{AGRADECIMIENTOS}

Este estudio, se desarrolló con el apoyo de la Dirección de Investigaciones (DIN) de la Universidad Pedagógica y Tecnológica de Colombia, en el marco del plan de trabajo del grupo de investigación Ecofisiología Vegetal, adscrito al programa de Ingeniería Agronómica de la Facultad de Ciencias Agropecuarias.

\section{BIBLIOGRAFÍA}

ASTEGIANO, E.D.; FAVARO, J.C.; BOUZO, C.A. 2001. Estimación del área foliar en distintos cultivares de tomate (Lycopersicum esculentum Mill.) utilizando medidas foliares lineales. Investigación Agraria: Producción y Protección Vegetales 16:249-256.

BLANCO, F.F.; FOLEGATTI, M.V. 2005. Estimation of leaf area for greenhouse cucumber by linear measurements under salinity and grafting. Sci. Agric. (Piracicaba, Braz.) 62(4):305-309.

BURTON, A.J., PREGITZER, K.S.; REED, D.D. 1991. Leaf area and foliar biomass relationships in northern hardwood forest located along an 80 acid deposition gradient. Forest Sci. 37(4):1041-1059.

CAMPOSTRINI, E.; YAMANISHI, O.K. 2001. Estimation of papaya leaf area using the central vein length. Scientia Agricola 58(1):39-42.

CANNELL, M.G.R.; MILNE, R.; SHEPPARD, L.J.; UNSWORTH, M.H. 1997. Radiation interception and productivity of willow. J. Appl. Ecol. 24:261-278.

ÇELIK, H.; UZUN, S. 2002. Validation of leaf area estimation models (Uzçelik-1) evaluated for some horticultural plants. Pakistan J. Botany, 34(1):41-46.

DEMRSOY, H.; DEMRSOY, L.; ÖZTÜRK, A. 2005. Improved model for the non-destructive estimation of strawberry leaf area. Fruits. 60(1):69-73

EBERT, T.; DERKSEN, R. 2004. A geometric model of mortality and crop protection for insects feeding 
on discrete toxicant deposits. J. Econ. Entomol. 97(2):155-162

GUO, D. P.; SUN, Y. Z. 2001. Estimation of leaf area of stem lettuce (Lactuca sativa var Angustana) from linear measurements. Indian J. Agr. Sci. 71:483-486.

HALPERN, C B.; MILLER, E.A.; GEYER, M.A. 1996. Equations for predicting above-ground biomass of plant species in nearly sucessional forest of the western Cascade Range, Oregon. NW Sci. 70(4):306-320.

KOZLOWSKI, T.T.; KRAMER, P.J.; PALLARDY, S.G. 1991. The physiological ecology of woody plants. New York. Acad. Press. 657p.

LANDSBERG, J.J. 1986. Physiological ecology of forest production. Academic Press, San Diego - Ca. 354p.

LANDSBERG, J.J.; HINGSTON, F.J. 1996. Evaluating a simple radiation/dry matter conversion model using data from Eucalyptus globulus plantations in Western Australia. Tree Physiology 16:801-808.

LAVIGNE, M.V.; LUTHER, J.E.; FRANKLIN, S.E.; HUNT, E.R. JR. 1996. Comparing branch biomass prediction equations for Abies balsamea. Can. J. Forest Res. 26:611-616.

LIN, K.C.; MA, F.C.; TANG, S.L. 2001. Allometric equations for predicting the aboveground biomass of tree species in the Fushan forest. Taiwan J. Forest Sci. 16(3):143-151.

LINDER, S.; MCMURTRIE, R.E.; LANDSBERG J.J. 1985. Growth of Eucalyptus: A mathematical model applied to Eucalyptus globulus. En: Tigerstedt, P.M.A; Puttonen, P.; Koski, V. (Eds.) Crop physiology of forest tree. Helsinki. Dept. Plant Breeding, U. of Helsinki. p.117-126.

LU, H.Y.; LU, C. T..; WEI, M.L.; CHAN, L.F. 2004. Comparison of different models for nondestructive leaf area estimation in taro. Agronomy J. 96:448-453.
LÖVENSTEIN, H.M., BERLINER, P.R. 1993. Biometric relationships for non-destructive above-ground biomass estimations in young plantations of Acacia salicina Lindl. and Eucalyptus occidentalis Endl. New Forest. 7:255-273.

MINISTERIO DE AGRICULTURA Y DESARROLLO RURAL. 2004. I censo nacional de 10 frutas agroindustriales y promisorias. Minagricultura, Colombia. 307p.

OLA-ADAMS, B.A. 1997. Asessment for three allometric regretion techniques of biomass determination in two hardwood species. J. Trop. Forest Sci. 9(3):321-328.

O'NEAL, M.; LANDIS, D.; ISAACS, R. 2002. An inexpensive, accurate method for measuring leaf area and defoliation through digital image analysis. J. Econ. Entomol. 95(6):1190-1194.

PRASADA RAO, G.S.; KHAN, B.H.; CHADHA, K.L. 1978. Comparison of methods of estimating leaf-surface area through leaf characteristics in some cultivars of Mangifera indica. Scientia Horticulturae. 8:341-348.

ROBBINS, N.S.; PHARR, D.M. 1987. Leaf area prediction models for cucumber from linear measurements. HortScience 22(6):1264-1266.

SEPASKHAH, A.R. 1977. Estimation of individual and total leaf areas of safflowers. Agronomy J. 69:783-785.

STRIK, B.C.; PROCTOR, J.T.A. 1985. Estimating the area of trifoliolate and unequally imparipinnate leaves of strawberry. HortScience. 20:1072-1074.

WARNOCK, R.; VALENZUELA, J.; TRUJILLO, A.; MADRIZ, P.; GUTIÉRREZ, M. 2006. Área foliar, componentes del área foliar y rendimiento de seis genotipos de caraota. Agronomía Trop. 56(1):21-42.

Recibido: Noviembre 30 de 2007

Aceptado: Abril 22 de 2008 